\title{
A Gradient Flow Approach to Robust Pole Assignment in Second-Order Systems
}

\author{
H. C. Chan \\ Department of Mathematics \\ City University of Hong Kong, Hong Kong \\ mahcchanosobolev.cityu.edu.hk
}

\author{
James Lam \\ Department of Mechanical Engineering \\ University of Hong Kong, Hong Kong \\ jlam@hkuxa.hku.hk
}

Daniel W. C. Ho

Department of Mathematics

City University of Hong Kong, Hong Kong

madanie10cityu. edu.hk

\begin{abstract}
Robust pole assignment for second-order systems is considered. It is shown that assignment can be achieved by solving a linear matrix equation or linear system. An objective function measuring the robustness of the closed-loop spectrum is minimized via gradient flow.
\end{abstract}

\section{Introduction}

Consider a time-invariant, second-order system

$$
M \ddot{q}+D \dot{q}+K q=f
$$

where $q, f \in \mathbb{R}^{n}$ and $M, D, K \in \mathbb{R}^{n \times n}$. Responses of (1) can be altered by applying a feedback control force $f=B u$ to (1) with $B \in \mathbb{R}^{n \times m}$ denoting the input matrix and $u \in \mathbb{R}^{m}$ the control vector. The state feedback control law is defined as $u=-F_{K} q-$ $F_{D} \dot{q}$ where $F_{D}$ and $F_{K}$ are derivative and proportional feedback matrices respectively. Hence, the resulting closed-loop system is

$$
M \ddot{q}+\left(D+B F_{D}\right) \dot{q}+\left(K+B F_{K}\right) q=0 .
$$

In this note, $M$ is assumed to be invertible and (2) can be written in the familiar first-order form

$$
\dot{z}=\bar{A} z+\bar{B} u
$$

where

$$
\bar{A}=\left[\begin{array}{cc}
0 & I \\
-M^{-1} K & -M^{-1} D
\end{array}\right], \quad \bar{B}=\left[\begin{array}{c}
0 \\
M^{-1} B
\end{array}\right] .
$$

For complete assignability, the pair $(\bar{A}, \bar{B})$ is assumed to be completely controllable.

\section{Pole Assignment}

Let $\Lambda$ be a real pseudo-diagonal matrix containing all the assigned poles, in which $1 \times 1$ blocks represent real poles and $2 \times 2$ blocks of the form

$$
\left[\begin{array}{cc}
\sigma & \omega \\
-\omega & \sigma
\end{array}\right]
$$

represent complex conjugate pairs $\sigma \pm i \omega$. Note that (3) is unitary equivalent to a diagonal matrix, that is

$$
U^{*}\left[\begin{array}{cc}
\sigma & \omega \\
-\omega & \sigma
\end{array}\right] U=\left[\begin{array}{cc}
\sigma+i \omega & 0 \\
0 & \sigma-i \omega
\end{array}\right]
$$

where $U=\frac{1}{\sqrt{2}}\left[\begin{array}{cc}1 & 1 \\ i & -i\end{array}\right]$ is a unitary matrix.

The idea of state feedback pole assignment is to find $\left[\begin{array}{ll}F_{K} & F_{D}\end{array}\right]$ such that

$$
\bar{A}+\bar{B}\left[\begin{array}{ll}
F_{K} & F_{D}
\end{array}\right]=T \Lambda T^{-1} \text { or } \bar{A}+\bar{B} G=T \Lambda
$$

where $G=\left[\begin{array}{ll}F_{K} & F_{D}\end{array}\right] T$ and $T$ is some nonsingular matrix. In fact, (4) corresponds to the following linear matrix equation

$$
\tilde{A} T \tilde{B}-\tilde{C} T \tilde{D}=\tilde{E}
$$

where

$$
\begin{gathered}
\tilde{A}=\left[\begin{array}{cc}
0 & I \\
-K & -D
\end{array}\right], \quad \tilde{B}=I, \quad \tilde{C}=\left[\begin{array}{cc}
I & 0 \\
0 & M
\end{array}\right], \\
\tilde{D}=\Lambda, \quad \tilde{E}=\left[\begin{array}{l}
0 \\
B
\end{array}\right]\left[\begin{array}{ll}
F_{K} & F_{D}
\end{array}\right] T=\left[\begin{array}{c}
0 \\
B
\end{array}\right] G .
\end{gathered}
$$

Since the pencils $\tilde{A}-\lambda \tilde{B}$ and $\tilde{D}-\lambda \tilde{B}$ are regular and the spectra $\rho(\tilde{A}, \tilde{C})$ and $\rho(\tilde{D}, \tilde{B})$ are assumed to be disjoint, i.e. no common closed-loop and open-loop poles, (5) is uniquely solvable for a given $G[1,3]$. However, by exploiting the special structures 
of the coefficient matrices $\tilde{A}, \tilde{B}, \tilde{C}, \tilde{D}, \tilde{E}$, we can express (5) into linear systems of equations which can then be solved by some effective algorithms [4]. Define $\hat{U}=\operatorname{diag}\left\{\hat{U}_{1}, \ldots, \hat{U}_{n+\frac{p}{2}}\right\}$ where $p$, an even number, is the number of real poles of (2) and

$$
\begin{cases}\hat{U}_{j}=1, & j=1, \ldots, p \\ \hat{U}_{j}=U, & j=p+1, \ldots, n+\frac{p}{2}\end{cases}
$$

Note that $\hat{U}=U \otimes I_{n}$ if $p=0$ and $\hat{U}=I_{2 n}$ if $p=2 n$. By comparing both sides of (5), we eventually obtain the linear system

$$
\left(\lambda_{j}^{2} M+\lambda_{j} D+K\right) w_{j}=-B \hat{g}_{j}, \quad j=1, \ldots, 2 n .
$$

where $\hat{g}$ is the $j$-th column of $\hat{G}=G \hat{U}$. Then for a given $G$, we can solve for $W=\left[w_{1}, \ldots, w_{2 n}\right]$ from (6) and $T=\left[\begin{array}{c}W \hat{U}^{*} \\ W \hat{U}^{*} \Lambda\end{array}\right]$. Consequently,

$$
\left[\begin{array}{ll}
F_{K} & F_{D}
\end{array}\right]=G T^{-1}
$$

which is the augmented derivative and proportional feedback matrix such that the closed-loop system (2) have the assigned spectrum.

\section{Robust Pole Assignment}

In measuring the robustness of the closed-loop spectrum, an objective function is defined as

$$
\phi(T(G))=\|T(G)\|_{F}^{2}+\left\|T(G)^{-1}\right\|_{F}^{2} .
$$

It can be shown that [5] the objective function (8) has to be minimized in order to obtain a robust closed-loop system. For notational simplicity, we write $\phi(T(G))$ as $\phi(G)$ throughout.

For a given $G, T$ is solved via the linear matrix equation (5) or linear system (6) at each minimization iteration for $\phi(G)$. When an optimum solution, say $G^{*}$, is reached such that $\phi\left(G^{*}\right)$ attains its minimum, the required augmented feedback matrix $F=\left[\begin{array}{ll}F_{K} & F_{D}\end{array}\right]=$ $G^{*} T^{*-1}$ is recovered from (7), where $G^{*}$ and $T^{*}$ satisfy (4).

With gradient flow analysis, this minimization problem comes down to solving the following system of ordinary differential equations

$$
\begin{aligned}
& \dot{G}(t)=2\left[\operatorname{trace}\left\{\frac{\partial T}{\partial g_{j k}}\left(T^{-1} T^{-T} T^{-1}-T^{T}\right)\right\}\right]_{m \times 2 n} \\
& G(0)=G_{0} \in \mathcal{G} .
\end{aligned}
$$

where $\mathcal{G}=\{G \mid T$ is a nonsingular solution of (4) $\}$ is open and dense in $\mathbb{R}^{m \times 2 n}$. The solution, say $G^{*}$, to (9) will be a minimum of $\phi(G)$ in (8). Important issues on the existence and convergence of the solution to (9) on $[0, \infty)$ are discussed in $[5]$.

In the ODE (12), $T=S \hat{U}^{*}$ where

$$
s_{l}=-\left[\begin{array}{c}
I \\
\lambda_{l} I
\end{array}\right] \mathcal{C}_{l}^{-1} B \hat{g}_{l}
$$

with $\mathcal{C}_{l}=\lambda_{l}^{2} M+\lambda_{l} D+K$. And also $\frac{\partial T}{\partial g_{j k}}=\frac{\partial S}{\partial g_{j k}} \hat{U}^{*}$ where

$$
\frac{\partial s_{l}}{\partial g_{j k}}=\left\{\begin{array}{c}
-\delta_{l k}\left[\begin{array}{c}
I \\
\lambda_{l} I
\end{array}\right] \mathcal{C}_{l}^{-1} b_{j} \\
k=1, \ldots, p \\
-\frac{\delta_{l k}+\delta_{l(k+1)}}{\sqrt{2}}\left[\begin{array}{c}
I \\
\lambda_{l} I
\end{array}\right] \mathcal{C}_{l}^{-1} b_{j} \\
k=p+1, p+3, \ldots, 2 n-1 \\
-i \frac{\delta_{l(k-1)}-\delta_{l k}}{\sqrt{2}}\left[\begin{array}{c}
I \\
\lambda_{l} I
\end{array}\right] \mathcal{C}_{l}^{-1} b_{j} \\
k=p+2, p+4, \ldots, 2 n
\end{array}\right.
$$

where $\delta_{j k}$ the Kronecker delta. It is important to realize that $\frac{\partial T}{\partial g_{j k}}$ is a constant matrix and is only required to evaluate once in the computation process.

\section{Conclusion}

In this note, the problem of robust pole assignment for second-order systems by state feedback is examined. It has been shown that pole assignment can be achieved by either solving a linear matrix equation or linear system. The assigned spectrum is made optimally robust by minimizing an objective function via gradient flow which involves the solution of a system of ordinary differential equation.

\section{References}

[1] K.-W. E. Chu, "The solution of the matrix equations $A X B-C X D=E$ and $(Y A-D Z, Y C-B Z)=$ (E,F)", Lin. Alg. Appl., 93, pp. 93-105 (1987).

[2] B. N. Datta and F. Rincón, "Feedback stabilization of a second-order system: a nonmodal approach", Lin. Alg. Appl., 188/189, pp. 135-161 (1993).

[3] G. D. Gardiner, A. J. Laub, J. J. Amato and C. B. Moler, "Solution of the Sylvester equation $A X B^{T}+$ $C X D^{T}=E ", A C M$ Trans. Math. Soft., 18, pp. 223231 (1992).

[4] G. H. Golub and C. F. Van Loan, Matrix Computations, 2nd Ed., Johns Hopkins University Press, Baltimore, 1989.

[5] J. Lam and W. Y. Yan, "A gradient flow approach to robust pole-placement problem", Internat. J. Robust Nonlinear Contr., 5, pp. 175-185 (1995). 